PROCEEDINGS OF THE

AMERICAN MATHEMATICAL SOCIETY

Volume 131, Number 11, Pages 3441-3446

S 0002-9939(03)06903-X

Article electronically published on February 6, 2003

\title{
LINEAR MAPS PRESERVING IDEALS OF C*-ALGEBRAS
}

\author{
JIANLIAN CUI AND JINCHUAN HOU
}

(Communicated by Joseph A. Ball)

\begin{abstract}
We show that every unital linear bijection which preserves the maximal left ideals from a semi-simple Banach algebra onto a $\mathrm{C}^{*}$-algebra of real rank zero is a Jordan isomorphism. Furthermore, every unital self-adjoint linear bijection on a countably decomposable factor von Neumann algebra is maximal left ideal preserving if and only if it is a *automorphism.
\end{abstract}

\section{INTRODUCTION}

Roughly speaking, linear preserver problems concern the characterization of linear maps between operator algebras that leave certain properties of elements invariant. Over the past decades much work has been done on linear preserver problems on matrix algebras. Recently, interest in similar questions on operator algebras over infinite dimensional spaces has also been growing. Particularly, the problem of characterizing linear maps which preserve the ideals was also considered (see [5], [7], [9]-[11], [13]).

Recall that a map $\Phi$ from a $\mathrm{C}^{*}$-algebra $\mathcal{A}$ into another preserves the maximal left ideals if $\Phi(\mathcal{J})$ is a maximal left ideal whenever $\mathcal{J}$ is; $\Phi$ preserves the maximal left ideals in both directions if $\Phi(\mathcal{J})$ is a maximal left ideal if and only if $\mathcal{J}$ is; $\Phi$ is self-adjoint if $\Phi\left(A^{*}\right)=\Phi(A)^{*}$ for every $A \in \mathcal{A}$.

The following two results were obtained in [10]:

Theorem A ([10, Theorem 2.3]). Let $\mathcal{A}$ be a finite simple von Neumann algebra acting on a separable Hilbert space $H$. If $\Phi: \mathcal{A} \rightarrow \mathcal{A}$ is a unital self-adjoint linear bijection that preserves the maximal left ideals in both directions, then there exists a unitary operator $U: H \rightarrow H$ such that $\Phi(A)=U A U^{*}$ for every $A \in \mathcal{A}$.

Theorem B $\left(\left[10\right.\right.$ Theorem 2.5]). Let $\mathcal{A}$ be a unital abelian $C^{*}$-algebra and let $\Phi: \mathcal{A} \rightarrow \mathcal{A}$ be a linear bijection that maps the maximal ideals to maximal ideals. Then there is an invertible element $V \in \mathcal{A}$ such that $\Phi(A B)=V \Phi(A) \Phi(B)$ for all $A, B \in \mathcal{A}$.

In this paper, we mainly discuss the linear maps on $\mathrm{C}^{*}$-algebras that preserve the maximal left ideals or maximal ideals in one direction or both directions, and generalize the results in [10] stated above by a different approach. We show that

Received by the editors November 7, 2001 and, in revised form, May 27, 2002.

2000 Mathematics Subject Classification. Primary 47B48, 47L30, 47A10.

Key words and phrases. Jordan homomorphism, maximal left ideals, Banach algebras, $\mathrm{C}^{*}$ algebras.

This work was supported by NNSFC and PNSFS. 
every unital linear bijection which preserves the maximal left ideals from a semisimple Banach algebra onto a $\mathrm{C}^{*}$-algebra of real rank zero is a Jordan isomorphism. Furthermore, every unital self-adjoint linear bijection on a countably decomposable factor von Neumann algebra is maximal left ideal preserving if and only if it is a *-automorphism.

\section{MAIN RESUlts AND PROOFS}

Let $\mathcal{A}$ and let $\mathcal{B}$ be unital Banach algebras and $T \in \mathcal{A}$. We denote by $\sigma(T)$, $\sigma_{l}(T), \partial \sigma(T)$ and $r(T)$ the spectrum, the left spectrum, the boundary of spectrum and the spectral radius of $T$, respectively; $\eta \sigma(T)$ stands for the polynomial convex hull of $\sigma(T)$. A linear map $\Phi: \mathcal{A} \rightarrow \mathcal{B}$ is left spectrum compressing (resp., spectrum compressing) if $\sigma_{l}(\Phi(A)) \subseteq \sigma_{l}(A)$ (resp., $\sigma(\Phi(A)) \subseteq \sigma(A)$ ) for every $A \in \mathcal{A}$; $\Phi$ is a Jordan homomorphism if the equation $\Phi\left(A^{2}\right)=\Phi(A)^{2}$ holds for all $A \in \mathcal{A}$; $\Phi$ is idempotent preserving if $\Phi$ maps idempotents into idempotents. The proof of the first lemma can be found in [2].

Lemma 1. Let $\mathcal{A}$ be a unital complex semi-simple Banach algebra. Then an element $P \in \mathcal{A}$ is an idempotent if and only if $\sigma(P) \subseteq\{0,1\}$ and there are positive numbers $r$ and $c$ such that $\sigma(T) \subseteq \sigma(P)+c\|P-T\|$ whenever $\|P-T\|<r$, where the set $\sigma(P)+c\|P-T\|$ denotes the union of the circular disks centered at points of $\sigma(P)$ with radius $c\|P-T\|$.

The following lemma was proved in [4]. For the sake of completeness, we state it and sketch its proof here.

Lemma 2. Let $\mathcal{A}$ and $\mathcal{B}$ be two semi-simple complex Banach algebras and let $\Phi: \mathcal{A} \rightarrow \mathcal{B}$ be a linear surjection. If $\Phi$ is left spectrum compressing (resp., spectrum compressing), then $\Phi$ preserves idempotent elements.

Proof. Assume that $\Phi$ is left spectrum compressing; then $\Phi$ is spectral radius nonincreasing. By the assumption that $\mathcal{B}$ is semi-simple and $\Phi$ is surjective, we obtain, by applying [1. Theorem 5.5.2], that $\Phi$ is continuous. Hence $\operatorname{ker} \Phi$ is a closed linear subspace of $\mathcal{A}$. Let $\pi: \mathcal{A} \rightarrow \mathcal{A} / \operatorname{ker} \Phi$ be the quotient map. Thus $\Phi$ induces a continuous, bijective linear map $\widehat{\Phi}: \mathcal{A} / \operatorname{ker} \Phi \rightarrow \mathcal{B}$ determined by $\widehat{\Phi} \circ \pi=\Phi$. Consequently, there are two positive constants $\alpha$ and $\beta$ such that $\alpha\|\pi(T)\| \leq\|\widehat{\Phi}(\pi(T))\|=$ $\|\Phi(T)\| \leq \beta\|\pi(T)\|$ for all $T$, where $\|\pi(T)\|=\inf \{\|T-A\| \mid A \in \operatorname{ker} \Phi\}$. Let $P$ in $\mathcal{A}$ be any idempotent. Then $\sigma_{l}(P) \subseteq \sigma(P) \subseteq\{0,1\}$. Obviously we may assume $\Phi(P) \neq 0$. Then $\partial \sigma(\Phi(P)) \subseteq \sigma_{l}(\Phi(P)) \subseteq\{0,1\}$, so $\sigma(\Phi(P)) \subseteq \eta \sigma(\Phi(P)) \subseteq\{0,1\}$. By Lemma 1, there are $r, c>0$ such that $\sigma(T+A) \subseteq\{0,1\}+c\|P-T-A\|$ whenever $\|P-T-A\|<r$. Suppose $T$ is given so that $\|\pi(P-T)\|<r$. For any sufficiently small $\varepsilon>0$, there is an element $A$ in $\operatorname{ker} \Phi$ such that $\|P-T-A\|<$ $\|\pi(P-T)\|+\varepsilon<r$. Thus we have, when $\|\pi(P-T)\|<r$,

$$
\sigma_{l}(\Phi(T)) \subseteq \sigma_{l}(T+A) \subseteq\{0,1\}+c\|\pi(P-T)\|+c \varepsilon .
$$

Letting $\varepsilon \rightarrow 0$, we get

$$
\sigma_{l}(\Phi(T)) \subseteq\{0,1\}+c\|\pi(P-T)\| \subseteq\{0,1\}+\frac{c}{\alpha}\|\Phi(P)-\Phi(T)\| .
$$

It follows that

$$
\sigma(\Phi(T)) \subseteq\{0,1\}+\frac{c}{\alpha}\|\Phi(P)-\Phi(T)\|
$$


for $\|\pi(P-T)\|<r$. Again, since $\widehat{\Phi}$ is an open surjection, there is an $r_{1}>0$ such that $\sigma(B) \subseteq\{0,1\}+\frac{c}{\alpha}\|\Phi(P)-B\|$, whenever $\|\Phi(P)-B\|<r_{1}$. Therefore, by Lemma 1 again, we have $\Phi(P)^{2}=\Phi(P)$, whence $\Phi$ preserves idempotents.

The argument above is still valid if $\Phi$ is spectrum compressing. The proof is completed.

Now, applying Lemma 2, we characterize the linear maps preserving the maximal left ideals from semi-simple complex Banach algebras onto $\mathrm{C}^{*}$-algebras of real rank zero. Note that every $\mathrm{C}^{*}$-algebra is semi-simple. To see this, assume that $Z$ is in the radical of a $\mathrm{C}^{*}$-algebra; then so is $Z Z^{*}$. Hence $Z Z^{*}$ is a self-adjoint element with spectrum $\{0\}$, consequently $Z Z^{*}=0$ and therefore $Z=0$. Recall that a $\mathrm{C}^{*}$ algebra $\mathcal{A}$ is of real rank zero if the set of all real linear combinations of orthogonal self-adjoint idempotents is dense in the set of all self-adjoint elements of $\mathcal{A}$ ([3]). It is clear that every von Neumann algebra is a $\mathrm{C}^{*}$-algebra of real rank zero. The following theorem is one of the main results in this note.

Theorem 3. Let $\mathcal{A}$ be a unital semi-simple complex Banach algebra and $\mathcal{B}$ a unital $C^{*}$-algebra of real rank zero. Suppose $\Phi: \mathcal{A} \rightarrow \mathcal{B}$ is a unital linear bijection. If $\Phi$ preserves the maximal left ideals, then $\Phi$ is a Jordan isomorphism. Furthermore, if one of $\mathcal{A}$ and $\mathcal{B}$ is prime, then $\Phi$ is either an isomorphism or an anti-isomorphism.

Proof. Note that an element is not left invertible if and only if it belongs to some maximal left ideal. Assume that $\Phi$ preserves the maximal left ideals. Since $\Phi$ is bijective and unital, we see that $\Phi^{-1}$ is unital and preserves left invertibility. It now follows that $\Phi^{-1}$ is left spectrum compressing, and hence idempotents preserving by Lemma 2 .

Pick a self-adjoint element $B$ in $\mathcal{B}$ which is a real linear combination of orthogonal self-adjoint idempotents, i.e., $B=\sum_{i=1}^{n} t_{i} P_{i}$ with $t_{i} \in \mathbb{R}, P_{i}^{2}=P_{i}=P_{i}^{*}$ and $P_{i} P_{j}=$ 0 if $i \neq j$. By Lemma $2, \Phi^{-1}$ is continuous and maps mutually orthogonal selfadjoint idempotents to mutually orthogonal idempotents. So $\Phi^{-1}\left(B^{2}\right)=\Phi^{-1}(B)^{2}$. Now, since $\Phi^{-1}$ is continuous and $\mathcal{B}$ is a $\mathrm{C}^{*}$-algebra of real rank zero, we see that $\Phi^{-1}\left(B^{2}\right)=\Phi^{-1}(B)^{2}$ holds for all self-adjoint elements $B$ in $\mathcal{B}$. Replacing $B$ by $C+D$, where both $C$ and $D$ are self-adjoint, we get $\Phi^{-1}(C D+D C)=$ $\Phi^{-1}(C) \Phi^{-1}(D)+\Phi^{-1}(D) \Phi^{-1}(C)$. Since every $T \in \mathcal{B}$ can be written in the form $T=$ $C+i D$ with $C$ and $D$ being self-adjoint, the last relation implies that $\Phi^{-1}\left(T^{2}\right)=$ $\Phi^{-1}(T)^{2}$. Hence $\Phi^{-1}$, as well as $\Phi$, is a Jordan isomorphism.

The last assertion of the theorem is obvious because it is well known (for example, see [6, pp. 47-51]) that every ring Jordan homomorphism from a ring onto a prime ring is either a ring homomorphism or a ring anti-homomorphism. This completes the proof.

Remark 4. If we replace the assumption $\Phi(I)=I$ by $\Phi(I)=D$ with $D$ being invertible in Theorem 3 , and let $L_{D}$ denote the map defined by $L_{D} T=D T$, then a small modification of the proof of Theorem 3 yields that $\Phi$ has the form $\Phi=$ $\Psi \circ L_{D^{-1}}$, where $\Psi$ is a Jordan isomorphism.

When $\Phi$ is a linear surjection preserving the maximal left ideals in both directions, we have

Corollary 5. Let $\mathcal{A}$ be a unital $C^{*}$-algebra of real rank zero and $\mathcal{B}$ a unital semisimple complex Banach algebra. Suppose $\Phi: \mathcal{A} \rightarrow \mathcal{B}$ is a linear surjection and $\Phi(I)$ 
is invertible. If $\Phi$ preserves the maximal left ideals in both directions, then $\Phi$ is a Jordan isomorphism multiplied from the left side by an invertible element.

Proof. Define $\Psi(T)=\Phi(I)^{-1} \Phi(T)$ for all $T \in \mathcal{A}$. Then $\Psi(I)=I$ and $\Psi$ preserves the maximal left ideals in both directions. We claim that $\Psi$ is injective. Let $T \in \mathcal{A}$ and $\Psi(T)=0$. Note that $\Psi$ preserves the left spectrum, so, for an arbitrary quasi-nilpotent $A \in \mathcal{A}, \sigma_{l}(T+A)=\sigma_{l}(\Psi(T+A))=\sigma_{l}(\Psi(A))=\sigma_{l}(A)=\{0\}$, hence $r(T+A)=0$. It follows from [1] that $T \in \operatorname{rad}(\mathcal{A})$, where $\operatorname{rad}(\mathcal{A})$ denotes the Jacobson radical of $\mathcal{A}$. This implies that $T=0$ since $\mathcal{A}$ is semi-simple. Now by Theorem $3, \Psi$ is a Jordan isomorphism and therefore $\Phi=L_{\Phi(I)} \circ \Psi$ is a Jordan isomorphism multiplied from the left side by an invertible element.

Let $\mathcal{A}$ be a von Neumann algebra and $P \in \mathcal{A}$ be a projection. Recall that $P$ is said to be countably decomposable relative to $\mathcal{A}$ when each orthogonal family of non-zero subprojections of $P$ in $\mathcal{A}$ is countable. If identity element $I$ is countable decomposable relative to $\mathcal{A}$, we say that $\mathcal{A}$ is countable decomposable; $\mathcal{A}$ is a factor if its center consists of the multiple of the identity element.

Now we discuss the case that the algebras are countably decomposable factor von Neumann algebras. Notice that every simple von Neumann algebra is a factor and every finite factor is countably decomposable. The following theorem particularly generalizes Theorem A by omitting the assumption "simplicity", "in both directions" and "separability".

Theorem 6. Let $\mathcal{A}$ be a countably decomposable factor von Neumann algebra acting on a Hilbert space $H$. Suppose $\Phi: \mathcal{A} \rightarrow \mathcal{A}$ is a unital self-adjoint linear bijection. Then $\Phi$ preserves the maximal left ideals if and only if $\Phi$ is a ${ }^{*}$-automorphism of $\mathcal{A}$. Furthermore, if $\mathcal{A}$ is finite or type III or semi-finite with its commutant $\mathcal{A}^{\prime}$ properly infinite, then there exists a unitary operator $U \in \mathcal{B}(H)$ such that $\Phi(A)=U A U^{*}$ for every $A \in \mathcal{A}$.

Proof. We only need to prove the necessity. By Theorem $3, \Phi$ is a *-Jordan isomorphism of $\mathcal{A}$. Since $\mathcal{A}$ is a factor, it follows from [14, Theorem 3.3] that $\Phi$ is either a *-automorphism or a ${ }^{*}$-anti-automorphism. Next we show that the last case never occurs.

Assume, on the contrary, that $\Phi$ is an anti-automorphism and let $\mathcal{J}$ be a maximal left ideal of $\mathcal{A}$. Then $\Phi(\mathcal{J})$ is a maximal left ideal. Taking any $D \in \mathcal{J}$ and $A \in \mathcal{A}$, since $\Phi(D) \Phi(A)=\Phi(A D) \in \Phi(\mathcal{J})$ and $\Phi$ is surjective, $\Phi(\mathcal{J})$ is also a right ideal. Thus $\Phi(\mathcal{J})$ is a maximal (two-sided) ideal for each maximal left ideal $\mathcal{J}$ of $\mathcal{A}$. This is impossible if $\mathcal{A}$ is a finite factor or a type III factor, since in these cases $\mathcal{A}$ is simple [8, p. 442]. If $\mathcal{A}$ is of type $\mathrm{I}_{\infty}$ or $\mathrm{II}_{\infty}$, then $\mathcal{A}$ has a unique proper normclosed ideal, i.e., the norm closure $\mathcal{K}$ of the ideal of operators with finite range projection [8, p. 443]. This means that, for every maximal left ideal $\mathcal{J}$ in $\mathcal{A}$, one has $\Phi(\mathcal{J})=\mathcal{K}$. It follows from the injectivity of $\Phi$ that $\mathcal{K}$ is also the only maximal left ideal in $\mathcal{A}$. However, it is easy to find a left ideal which contains an element not belonging to $\mathcal{K}$ (for example, one can get examples from the unilateral shift). This completes the proof of the first assertion in the theorem.

As to the second assertion, note that every *-automorphism of a finite or type III or semi-finite with its commutant properly infinite factor von Neumann algebra is spacial [12, Propositions 2.9.24, 2.9.25, 2.9.26, pp.118-119]. So there exists a unitary operator $U \in \mathcal{B}(H)$ such that $\Phi(A)=U A U^{*}$ for every $A \in \mathcal{A}$. 
Corollary 7. Let $\mathcal{A}$ be a countably decomposable factor von Neumann algebra acting on a Hilbert space $H$. Suppose $\Phi: \mathcal{A} \rightarrow \mathcal{A}$ is a self-adjoint linear surjection and $\Phi(I)$ is invertible. If $\Phi$ preserves the maximal left ideals in both directions, then $\Phi$ is a *-automorphism of $\mathcal{A}$ multiplied from the left side by $\Phi(I)$.

We say a subset $\mathcal{J} \subset \mathcal{A}$ is a maximal semi-ideal if it is either a maximal left ideal or a maximal right ideal. In the sequel we discuss the linear maps which preserve the maximal semi-ideals.

Theorem 8. Let $\mathcal{A}$ be a unital semi-simple complex Banach algebra and $\mathcal{B}$ a unital $C^{*}$-algebra of real rank zero. Suppose $\Phi: \mathcal{A} \rightarrow \mathcal{B}$ is a linear bijection. If $\Phi$ preserves the maximal semi-ideals, then there exists a Jordan isomorphism $\Psi$ and an invertible element $D$ such that $\Phi=\Psi \circ L_{D}$.

Proof. Since an element fails to have an inverse if and only if it is included in a maximal semi-ideal, and since $\Phi$ is bijective, $\Phi^{-1}$ must preserve the invertibility of elements. Thus $\Phi^{-1}(I)$ is invertible in $\mathcal{A}$. Hence there is an invertible element $D$ such that $\Phi^{-1}(I)=D^{-1}$. Let $\varphi=L_{D^{\circ}} \Phi^{-1}$; then $\varphi: \mathcal{B} \rightarrow \mathcal{A}$ is a unital linear bijection compressing the spectrum. It follows from Lemma 2 and the proof of Theorem 3 that $\varphi$ is a Jordan isomorphism and $\Phi^{-1}=L_{D^{-1}} \circ \varphi$. Now it is clear that $\Phi=\Psi \circ L_{D}$ with $\Psi=\varphi^{-1}$ being a Jordan isomorphism.

To characterize the linear bijections preserving the maximal ideals between commutative Banach algebras, the following lemma is needed, of which the proof can be found in [5] and [9].

Lemma 9. Let $\mathcal{A}$ and $\mathcal{B}$ be two commutative Banach algebras containing unit elements, and suppose that $\mathcal{B}$ is semi-simple. If $\Phi: \mathcal{A} \rightarrow \mathcal{B}$ is a linear map compressing the spectrum, then $\Phi$ is a homomorphism.

The following result is a generalization of Theorem B.

Corollary 10. Let $\mathcal{A}$ and $\mathcal{B}$ be two unital commutative Banach algebras, and suppose that $\mathcal{A}$ is semi-simple. If $\Phi: \mathcal{A} \rightarrow \mathcal{B}$ is a linear bijection preserving the maximal ideals, then $\Phi$ is an isomorphism multiplied by an invertible element.

Proof. Since $\mathcal{A}$ and $\mathcal{B}$ are commutative and $\Phi$ is bijective, $\Phi$ preserves the maximal ideals if and only if $\Phi^{-1}$ preserves the invertibility. Let $\varphi=L_{D^{-1}} \circ \Phi^{-1}$, where $D=\Phi^{-1}(I)$; then $\varphi(I)=I$ and $\varphi: \mathcal{B} \rightarrow \mathcal{A}$ preserves the invertibility. So $\varphi$ compresses the spectrum, hence $\varphi$ is an isomorphism by Lemma 9. Let $\Psi=\varphi^{-1}$; it is clear that $\Psi$ is also an isomorphism. Denote the invertible element $\Psi\left(D^{-1}\right)$ by $E$; we get $\Phi(A)=\Psi \circ L_{D^{-1}}(A)=\Psi\left(D^{-1} A\right)=E \Psi(A)$ for every $A \in \mathcal{A}$, that is, $\Phi=L_{E} \circ \Psi$.

\section{ACKNOWLEDGMENTS}

This work was done during the second author's visit at the Institute of Mathematics, Academy of Mathematics and System Sciences, Chinese Academy of Sciences. He is grateful to the hospitality of Professor Li Bingren and Professor Ge Liming. 


\section{REFERENCES}

[1] Aupetit, B., A Primer On Spectral Theory, Springer, New York, 1991. MR 92c:46001

[2] Aupetit, B., Spectrum-preserving linear mappings between Banach algebras or JordanBanach algebras, J. London Math. Soc. (2) 62 (2000), No. 3, 917-924. MR 2001h:46078

[3] Brown, L.G., Pedersen, G.K., C*-algebras of real rank zero, J. Funct. Anal. 99 (1991) 131-149. MR 92m:46086

[4] Cui, J., Hou, J., Linear maps between semi-simple Banach algebras compressing certain spectral functions, Rocky Mountain J. Math., to appear.

[5] Gleason, A. M., A characterization of maximal ideals [J], J. Analyse Math. 19 (1967), 171-172. MR 35:4732

[6] Herstein, I.N., Topics in ring theory, Springer, Berlin, 1991.

[7] Johnson, B. E., Centralizers and operators reduced by maximal ideals, J. London Math. Soc., 43 (1968), 231-233. MR 36:6937

[8] Kadison, R. V. and Ringrose J. R., Fundamentals of the Theory of Operator Algebras II, GSM 16, AMS, 1997. MR 98f:46001b

[9] Kahane, J. P. and Zelazko, W., A characterization of maximal ideals in commutative Banach algebras [J], Studia Math. 29 (1968), 339-343. MR 37:1998

[10] Kim, S. O., Linear maps preserving ideals of $\mathrm{C}^{*}$-algebras, Proc. Amer. Math. Soc., Article electronically published on October 25, 2000. MR 2001m:47077

[11] Molnar, L., Some linear preserver problems on $\mathcal{B}(H)$ concerning rank and corank, Lin. Alg. Appl., 286 (1999), 311-321. MR 2000b:47089

[12] Sakai, S., C*-algebras and $W^{*}$-algebras, Springer Verlag, New York, 1971. MR 56:1082

[13] Shul'man, V. S., Operators preserving ideals in $C^{*}$-algebras, Studia Math., 109(1), (1994), 67-72. MR 95b:46097]

[14] Stormer, E., On the Jordan structure of $\mathrm{C}^{*}$-algebras, Trans. Amer. Math. Soc. 120 (1965), 438-447. MR 32:2930

School of Mathematical Sciences, Peking University, Beijing 100871, People's RePUBLIC OF CHINA

Current address: Department of Applied Mathematics, Taiyuan University of Technology, Taiyuan 030024, People's Republic of China; Department of Mathematics, Shanxi Teachers University, Linfen, 041004, People's Republic of China

E-mail address: cuijl@dns.sxtu.edu.cn

Department of Mathematics, Shanxi Teachers University, Linfen, 041004, People's Republic of China

E-mail address: jhou@dns.sxtu.edu.cn 